\title{
Histological and Ultrastructural Changes of Cardiomyocytes in Experimental Rats with Tail Thrombosis following Subplantar Application of Carrageenin
}

\author{
Rostislav Kod'ousek $^{a}$ Jaroslav Jezdínskýb Dimitrolos Krajčíc \\ a Institute of Pathology, ${ }^{b}$ Institute of Pharmacology, Faculty of Medicine, Palacky University, Olomouc, \\ Czech Republic, and 'Department of Anatomy, Faculty of Medicine, Kuwait University, Kuwait
}

\section{Key Words}

Carrageenin · Rat tail thrombosis $\cdot$ Mitochondrial

cardiomyopathy $\cdot$ Granular deposits

\begin{abstract}
Objective: To describe histological and ultrastructural changes of cardiomyocytes in experimental rats following subplantar administration of carrageenin. Material and Methods: In adult rats, an acute inflammatory reaction was induced by subplantar injection of $0.1 \mathrm{ml}$ of $1 \%$ sterile carrageenin solution. In a total of 10 rats, which developed gangrene of tails in 5- to 12-cm-long segments, were killed and their internal organs fixed in 10\% formaldehyde solution and subsequently processed for paraffin embedding. Later, blocks of the ventricular heart tissue were refixed and reprocessed for Araldite embedding and ultrastructure observation. Similarly, the cardiac muscle of control, carrageenin-injected rats which did not develop vascular thrombosis was processed. Results: The cardiomyocytes of rats injected with carrageenin showed focal dystrophic alterations, enlarged
\end{abstract}

Some of the results shown in this paper have been presented as a poster at the 10th Annual HSC Poster Day, Faculty of Medicine, Kuwait, April 2004.
(C) 2007 S. Karger AG, Basel

$1011-7571 / 07 / 0165-0360 \$ 23.50 / 0$

Fax +41613061234

E-Mail karger@karger.ch

www.karger.com
Accessible online at: www.karger.com/mpp mitochondria with densely packed concentrically oriented cristae, and many dense and irregularly shaped deposits with microgranular helicoid organization. Normal cardiomyocytes were observed in control rats. Complicating thrombosis of tail blood vessels leading to extensive tail necroses were also histologically confirmed. Conclusion: These findings demonstrate specific pathogenic effect in the cardiovascular system of the carrageenin-treated rats.

Copyright $\odot 2007$ S. Karger AG, Basel

\section{Introduction}

Carrageenins represent a family of polysaccharide polymers of lectin type extracted from various red seaweeds (Rhodophycae). Biochemically, they are high molecular sulphated polysaccharides - polygalactans [1]. Pharmaceutically, they belong to the group of Mucillagines, in which alternating units of $\alpha$ - and $\beta$-galactopyranose form linear, strongly anionic polymers with highly flexible molecules of molecular weight $>100,000$. Some carrageenins ( $\kappa$ and $\iota)$ at higher concentrations, wind around each other to form double-helical zones. On cooling, the helical formation of the molecules promotes gel formation (mainly k-carrageenin). On the other hand,

Dr. Krajčí Dimitrolos

Department of Anatomy, Faculty of Medicine

Kuwait University, P.O. Box 24923

Safat 13110 (Kuwait)

Tel. +965 5312300 6268, Fax +965 531 9478, E-Mail krajci@hsc.edu.kw 
$\lambda$-carrageenin, due to its structure of alternating monomeric units of D-galactose 2-sulphate and D-galactose 2,6-disulphate, does not form gel structure. Degradation of natural carrageenin produces low molecular weight poligeenan [2] with different biological properties.

In the food industry, food-grade carrageenin (E407) is used mainly for thickening, suspending and gelling, in preparing cooked meat, sausages and yoghurts. Although the European Commission of Scientific Committee on Food stated that food-grade carrageenin is safe to use [2, $3]$, some earlier evidence from animal studies has demonstrated that degraded carrageenin, with a molecular weight of $\leq 30,000$, causes ulcerations and malignancies in the gastrointestinal tract [4] and affects incidence of mammary carcinoma [5]. Carrageenin, which can in limited amounts pass through intestinal barrier in experimental animals, may be harmless in healthy organisms, but it may become toxic in groups with disorders of the gastrointestinal tract [1]. The adverse effects of poligeenan (a product of acid hydrolysis in stomach) are ulcerations of intestinal mucosa [6-8], or stimulating neoplastic effect [9]. Ishioka et al. [10] have reported a variety of changes ranging from the initial intestinal inflammations, through ulcerative lesions and squamous metaplasia, to colorectal tumors in experimental rats. Carcinogenesis in the mammary gland was reported by Tobacman [11] and Tobacman and Walters [12] as an effect of poligeenan or $\lambda$-carrageenin on mammary myoepithelial cells grown in tissue culture, but Zhou et al. [13] reported an antitumorous effect of $\lambda$-carrageenin of various molecular weight.

When administered systemically (parenterally) to experimental animals, carrageenins feature agglutinating activity against blood cells and also against transformed cells in tissue culture [14], mitogenic properties and interaction with some receptors in cell membranes [15]. In general, lectins bind to the saccharide residues of membrane receptors and this reaction can be visualized with a labeled lectin, or it can result in a visible agglutination of cells. Lectins can be used for agglutination of erythrocytes within the range of $\mathrm{ABO}$ blood group system, whereas some special lectin fractions show increased hemaglutinating activity [16]. Thomson et al. [17] have shown that intravenous administration of carrageenins to experimental animals developed disseminated coagulopathy. Bekemeier et al. $[18,19]$ and Hirschelmann and Bekemeier [20] reported thromboses of tail blood vessels in experimental rats after administration of $\kappa$-carrageenin. This effect has been later used by Bertelli et al. [21] for testing of antithrombotics.

Ultrastructure of Cardiomyocytes in

Carrageenin-Injected Rats
An effect of carrageenin on the immune system has also been observed, namely a selective toxic effect on antigen-processing macrophages, humoral and cellular immunity [22] and marked suppression of the immune system [23].

In experimental pharmacology research, when testing various anti-inflammatory drugs, the subplantar application of carrageenin solution is widely used (doses of $500-1,000 \mu \mathrm{g}$ in rat and $300 \mu \mathrm{g}$ in mouse) to induce aseptic subcutaneous inflammation of paws. Local edematous swelling of the paw can be easily measured, and the anti-inflammatory effect of the investigated drug objectively evaluated [24]. The edematogenic activity of carrageenin decreases with depolymeration, and with the fraction of molecular weight 73,700 and lower this effect is almost absent [25].

Because various adverse effects have been observed in carrageenin-treated rats as outlined above, we decided to investigate histological and ultrastructural changes of carrageenin-induced cardiotoxicity in rats that developed necrotic tails due to thrombosis of tail arteries and veins but also peculiar dystrophic changes in the myocardium.

\section{Material and Methods}

Female adult Wistar rats (Lab. Animal Farm, Konarovice, Czech Republic) weighing 200-250 g, bred under standard conditions, which developed tail necrosis within $24 \mathrm{~h}$ after subplantar injection of $0.1 \mathrm{ml}$ of $1 \%$ carrageenin solution in a sterile aqua pro inj. (Carrageenan Type I, commercial grade, Sigma, UK) were studied microscopically. In total, 10 rats out of 20, which developed gangrene of tails in the group of 50 injected experimental animals, were killed the next day under pentobarbital anesthesia. The tails, heart, lung, liver and kidney were removed and fixed in $10 \%$ formaldehyde and subsequently processed for paraffin embedding. Similarly, the organs of another 10 injected rats, which did not develop tail necroses, as well as 5 noninjected rats, were processed as controls. Paraffin sections were stained with standard hematoxylin and eosin method, with a modified Giemsa's method and with a Hale-Mueller's reaction for visualization of acid mucopolysaccharides, which is based on their binding to the colloidal iron (ferrihydroxidsol).

Later, blocks of the ventricular heart tissue were deparaffinized, rehydrated, and refixed with $3 \%$ glutaraldehyde for $2 \mathrm{~h}$ (SPI, Westchester, Pa., USA) and postfixed with $1 \%$ buffered osmium tetroxide (SPI) for $2 \mathrm{~h}$, prior to reprocessing for Araldite embedding (Araldite 502 resin kit, TAAB, Aldermaston, UK). Semithin sections stained with $1 \%$ toluidine blue in borax solution were used for light microscopic screening. Ultrathin sections, double-stained with uranyl acetate and lead citrate, were observed under the JEOL 1200EXII (JEOL, Tokyo, Japan) transmission electron microscope at $80 \mathrm{kV}$. 
Fig. 1. a An experimental rat developing a vascular thrombosis with gangrene of the tail $24 \mathrm{~h}$ after application of carrageenin. b Massive vascular thrombosis of the tail leading to subsequent necrosis. HE. $\times 54$.

Fig. 2. a Pronounced tinctorial abnormalities of cardiomyocytes with central achromophilic hyaline zones, visualized mainly in the modified Giemsa-cresyl violet stain in a carrageenin-injected rat. $\times 220$. b A semithin section of heart tissue in a carrageenin-injected rat. Note the peculiar mosaic-like appearance of the cytoplasm of cardiomyocytes in transverse sections. Toluidine blue stain. $\times 300$. c Myocardium of carrageenin-injected rat showing staining variability of fibers, slight rarefaction of myofibrils and focal accumulation of enlarged spherical mitochondria (arrows). Semithin section, toluidine blue stain. $\times 450$.
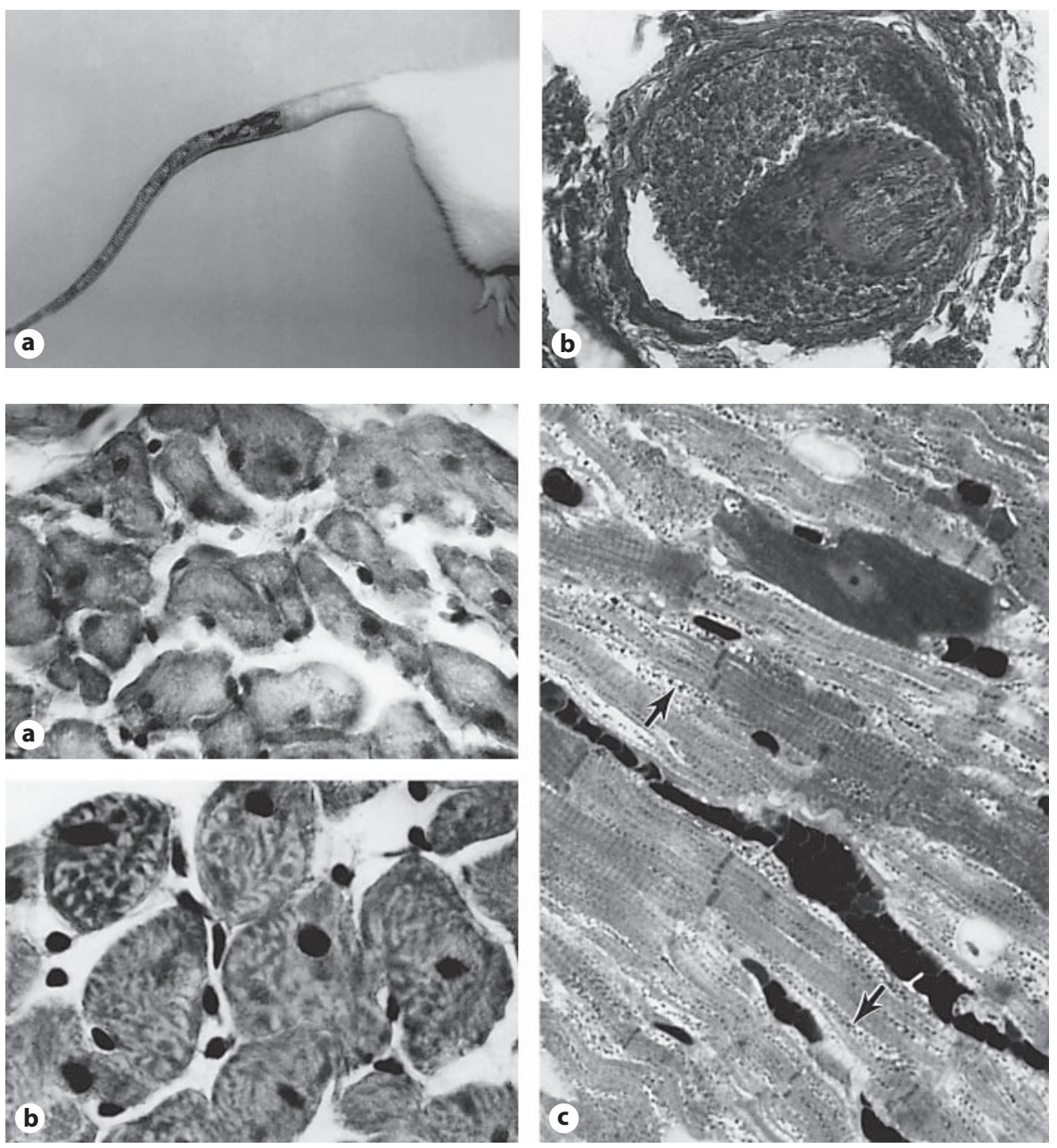

\section{Results}

Necroses of the tail (fig. 1a) with associated vascular thrombosis (fig. 1b) were observed. A positive HaleMueller reaction was demonstrable on the surface of erythrocytes in thrombi.

Changes were seen in the left ventricle showing focal dystrophic alteration of cardiomyocytes, with only minimal reactive cellulization in the interstitium (fig. $2 \mathrm{a}, \mathrm{b}$ ). These foci revealed staining irregularities, prominent swelling with hyaline appearance of cardiomyocytes, and in some places also visible granularity of the cytoplasm (fig. 2c). The above-mentioned staining irregularities were impressive in routine paraffin preparations stained with a modified Giemsa's method (fig. 2a) and in semithin sections stained with Toluidine blue (fig. 2b). These morphological findings permitted provisional diagnosis of carrageenin-induced cardiomyopathy.
In the ultrastructure, longitudinally oriented sections of cardiomyocytes of rats with tail necroses revealed striated myofibrils with signs of rarefaction and disorientation (fig. 3a). Interfibrillar spaces were wide. Prominent, moderately enlarged mitochondria (up to $1.6 \mu \mathrm{m}$ in length) accumulated near nuclear poles, subsarcolemmal, or were linearly arranged in between the myofibrils. In the interfibrillar locations, the enlarged, mostly spherical mitochondria did not match the length of sarcomeres (fig. 3a). Due to more spherical shapes of mitochondria, the mean area in sections increased from $0.32 \pm 0.09$ $\mu \mathrm{m}^{2}$ in controls to $0.51 \pm 0.19 \mu \mathrm{m}^{2}$ in carrageenin-injected rat. Mitochondrial matrix was always very dense and contained a high accumulation of densely-packed long mitochondrial cristae, which filled up almost completely the profile of this organelle. In some sections, the crowded mitochondrial cristae were arranged concentrically (fig. 3b), while in others, the mitochondria looked 
Fig. 3. a Ultrastructure of ventricular cardiomyocyte from carrageenin-injected rat with tail thrombosis displays marked rarefaction and disorientation of myofibrils. Wide interfibrillar spaces contain enlarged mitochondria (M) that no longer match the length of sarcomeres. Numerous dense granular deposits (arrows) spread among the mitochondria, tend to fuse into larger irregular units. $\times 6,000$. b High-resolution electron micrograph shows enlarged spherical mitochondria with long, densely packed and concentrically arranged cristae (asterisk) filling up the entire profile of the organelle. $\times 36,000$. c Irregular-shaped granular deposits (GD) of unspecified nature in interfibrillar spaces are not limited by a membrane. They do not contain any lamellar components either. $\times 45,000$. Inset Substructure of the granular deposition reveals its composition of tightly-packed subunits with helicoid organization. $\times 250,000$.
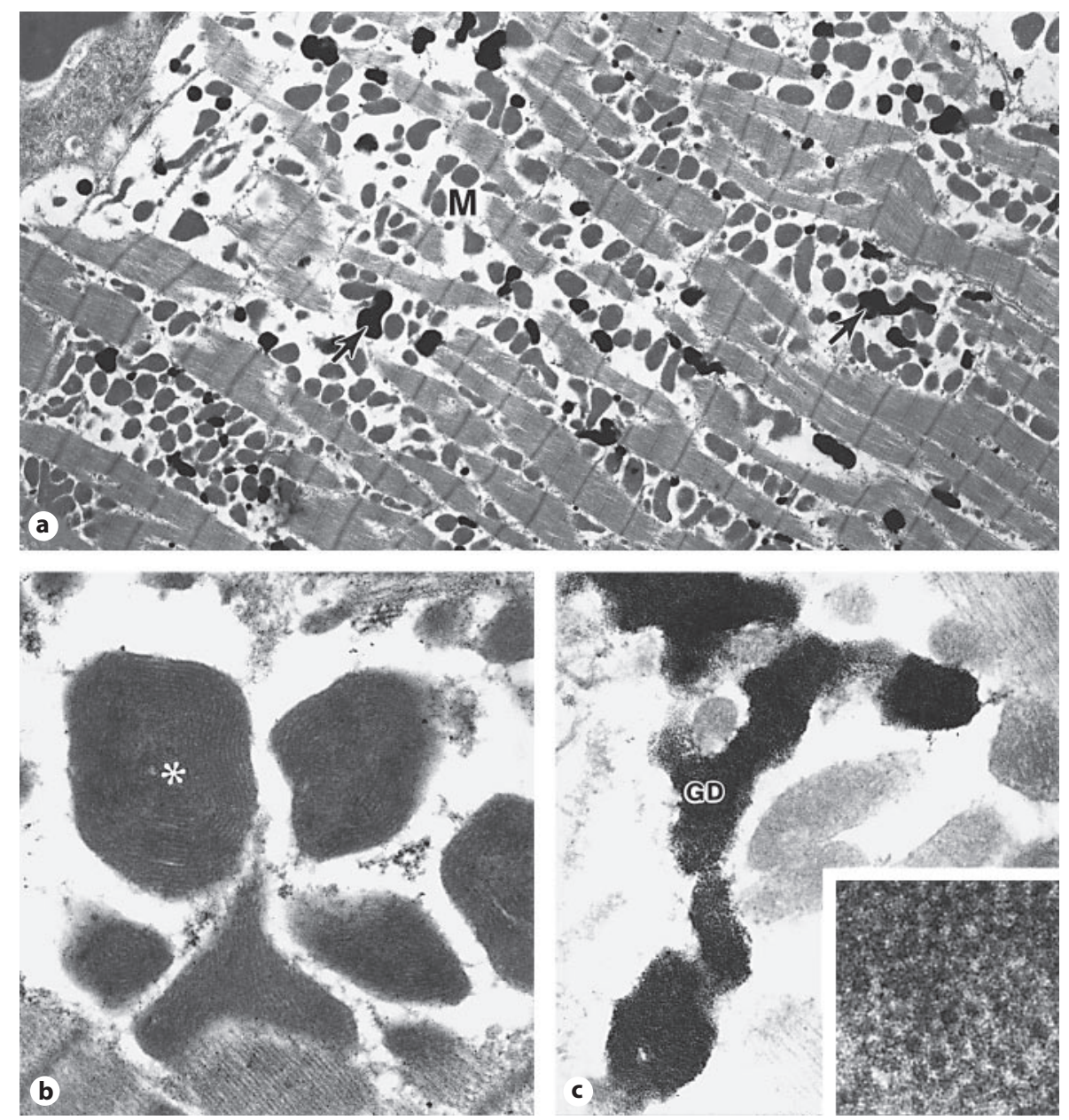

homogenously dense inside with no resolution of intracristal spaces.

Some cardiomyocytes also contained, small, dense and irregularly shaped granular-structured deposits located individually or in groups in between the mitochondria, with a tendency of fusing into irregularly shaped units (fig. 3a, c). These granular deposits were neither limited by a membrane unit nor contained any lamellar components inside. In these deposits, the high magnification electron micrographs revealed tightly packed microgranular subunits with helicoid organization (fig. 3c).

Normal cardiomyocytes in the control rats (fig. 4) showed continuous, regularly spaced myofibrils alternating with serially arranged, elongated mitochondria that were closely related to the length of sarcomeres and to transversally cut profiles of the sarcoplasmic reticulum with $\mathrm{T}$ tubules. These mitochondria contained transver- sally oriented cristae within a moderately dense mitochondrial matrix. The only location of grouped or ovalshaped mitochondria were in subsarcolemmal or paranuclear compartments of cardiomyocytes (fig. 4).

\section{Discussion}

The indicative lesion was the development of necroses in the rat tail caused by vascular thrombosis in a number of experimental rats. An early description of this phenomenon after intravenous or intraperitoneal injection of $\mathrm{\kappa}$-carrageenin in rats and also in mice was reported earlier by Bekemeier et al. $[19,26]$ and, in later years, it has been widely used as a model for testing antithrombotics $[18,20,21]$.

Tail necroses, due to the thrombosis of the tail blood vessels, in some of the experimental animals were likely 


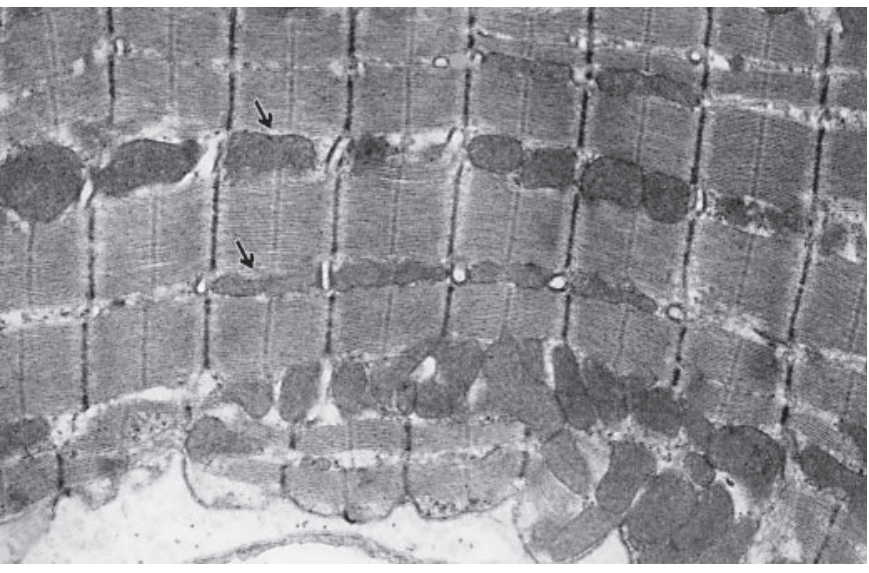

Fig. 4. Normal cardiomyocyte in a noninjected control rat shows continuous and regularly spaced myofibrils that alternate with serially arranged, elongated mitochondria (arrows). Interfibrillar mitochondria are closely related to the length of sarcomeres and to transversally cut profiles of the sarcoplasmic reticulum with $\mathrm{T}$ tubules. They contain transversally oriented cristae within a moderately dense mitochondrial matrix. $\times 12,000$.

caused by systemic resorption of a small amount of carrageenin injected into the paws, either by direct resorption or mediated transfer through lymphatic circulation. Based on our experience, we assume a great variability in the sensitivity of laboratory rats and mice to the thrombogenic effect of carrageenin, not only according to specific strains, but also due to seasonal variations. This is also supported by findings of Liang et al. [27], as they have been able to facilitate carrageenin-induced tail blood vessel thrombosis in rats $(25 \mathrm{mg} / \mathrm{kg}$, i.p.) and mice $(150 \mathrm{mg} /$ kg, i.p.) with intravenous application of endotoxin (LPS), which was to act as a sensibilizing agent to the thrombogenic effect of carrageenin.

Myopathic changes in cardiomyocytes, presumed in histological sections, were verified by electron microscopy showing disruption, displacement and rarefaction of myofibrils and enlargement and ultrastructural alterations of interfibrillar mitochondria. These ultrastructural features are analogous to some cases of mitochondrial myopathies as described in a variety of muscular disorders where abnormal mitochondria were also found [28, 29]. In myopathies, the mitochondrial changes are manifested by their enlargement up to $14 \mu \mathrm{m}$ in length (megamitochondria) and marked augmentation of the number and length of mitochondrial cristae which acquire zigzag, concentric or paracristalline appearance [28, 29]. In our study, the size of mitochondria did not exceed 1.6 $\mu \mathrm{m}$ in length, but most of them lost their regular align- ment along the myofibrillar sarcomeres and acquired more spherical shapes with approximately $50 \%$ increase in section area. This, together with the impressive increase in number and length of internal cristae and tight packing into concentric formations indicates deranged mitochondrial pattern in myocardial cells with subsequent metabolic abnormalities in rats with systemic reaction to carrageenin. Furthermore, a clear evidence of storage or persistence of the dense granular material was found in the interfibrillar spaces. Since these granular densities are not membrane limited, they were considered as inclusions rather than lysosome-derived structures. By the substructure, as shown in the inset to figure $3 c$, these granular deposits clearly differ from monoparticulate or aggregated glycogen deposits, which may also be found in the sarcoplasm of normal or pathological cardiomyocytes [29]. These accumulated deposits can also be responsible for pronounced tinctorial abnormalities of cardiomyocytes visualized in histological sections. The chemical nature of this deposited material and the route of its entry into cardiomyocytes remains yet to be elucidated. No ultrastructural signs of endocytosis of such dense material has been observed in the sampled cardiomyocytes. With regard to the high electron density and tight packing, the granular densities in cardiomyocytes differ from the carrageenins experimentally administered and then taken up and stored in macrophages $[30,31,32]$ and in some other cells, like mammary myoepithelial cells in tissue cultures [12], and Kupffer cells [33], where they have been observed as a homogenous, fibrillar, or only moderately dense granular material with occasional redundant internal lamellations, located in membrane-limited endosomes.

Interestingly, any sign of terminal damage to cardiomyocytes in terms of apoptosis or necrosis was not observed. This is in contrast to observations of Calkosinski et al. [34] who described apoptotic changes in myocardium of rats in the course of experimentally induced pleurisy, and explained their development with the action of released anti-inflammatory cytokines IL1, ILG and TNF- $\alpha$. In their experiment, the first myocardial reaction to the remote inflammatory process was an increase in mitochondrial volume within $24 \mathrm{~h}$, followed by derangement of the internal structure - the cristae - within $72 \mathrm{~h}$. Apoptotic nuclei were observed within $120 \mathrm{~h}$ of the inflammatory process [34]. No thrombotic complication was reported. We speculate that the apoptotic damage to cardiomyocytes observed in these experiments could be due to intravascularly penetrated carrageenin, as that could have been more easily resorbed from the pleural 
cavity within the given time course than from the intraplantar injection in rat paw, as in our experiments.

Although, we cannot specify the route of transfer of the injected carrageenin from the connective tissue of the hind paw to the systemic circulation and its uptake by cardiac muscle cells, we would like to point out that care should be taken when using the tissues from these injected animals for other pharmacology examinations even if the tissue is remote from the injection site. Furthermore, the pharmacological data on tested drugs obtained on such experimental models might be modified by alteration of remote organs like the heart. This is also supported by a wide range of hematological changes observed following intraperitoneal injection of carrageenins [35].

\section{Conclusion}

This study showed marked mitochondrial lesions in cardiomyocytes in rats following subplantar injection of carrageenin which were found in animals that simultane- ously developed thrombosis of tail blood vessels with subsequent tail necrosis. In these animals, storage or persistence of a dense granular material was also found in the interfibrillar spaces of cardiomyocytes. These documented structural changes of cardiomyocytes were not described earlier in relation to the thrombogenic effect of carrageenin in rats or other animals. In our opinion, the pathogenic effect of carrageenin in relation to the damage of cardiomyocytes should be given further attention.

\section{Acknowledgment}

The authors would like express their thanks to Mrs. Amal Wagdi, the Laboratory Technician at the Department of Anatomy, for her contribution to the methodical part of this project. Electron microscope has been provided by the Electron Microscopy Unit, Faculty of Medicine, KU, under the Shared Facility Project No. GM 01/01. Preliminary examination of this material has been also performed at the EM laboratory, Institute of Pathology in Olomouc, using TESLA BS-500 electron microscope.

\section{References}

$\checkmark 1$ Nicklin S, Miller K: Intestinal uptake and immunological effects of carrageenan - current concepts. Food Addit Contam 1989;6: 425-436.

2 Cohen SM, Ito N: A critical review of the toxicological effects of carrageenan and processed eucheuma seaweed on the gastrointestinal tract. Crit Rev Toxicol 2002;32: 413-444.

3 Weiner ML: Toxicological properties of carrageenan. Agents Actions 1991;32:46-51.

4 Tobacman JK: Review of harmful gastrointestinal effects of carrageenan in animal experiments. Environ Health Perspect 2001; 109:983-994

5 Tobacman JK, Wallace RB, Zimmerman MB: Consumption of carrageenan and other water-soluble polymers used as food additives and incidence of mammary carcinoma. Med Hypotheses 2001a;56:589-598.

6 Watt J, Marcus R: Harmful effects of carrageenan fed to animals. Cancer Detect Prev 1981;4:129-134.

7 Marcus SN, Marcus AJ, Marcus R, Ewen SW, Watt J: The pre-ulcerative phase of carrageenan-induced colonic ulceration in the guinea-pig. Int J Exp Pathol 1992;73:515526.
8 Yasuyuki O, Tomonori I, Kazuo W, Noriyuki $\mathrm{K}$ : A study on carcinogenesis induced by degraded carrageenan arising from squamous metaplasia of the rat colorectum. Cancer Lett 1981;14:267-272.

-9 Wilcox DK, Higgins J, Bertram TA: Colonic epithelial cell proliferation in a rat model of nongenotoxin-induced colonic neoplasia. Lab Invest 1992;67:405-411.

10 Ishioka T, Kuwabara N, Oohashi Y, Wakabayashi K: Induction of colorectal tumors in rats by sulfated polysaccharides. Crit Rev Toxicol 1987;17:215-244.

11 Tobacman JK: Filament disassembly and loss of mammary myoepithelial cells after exposure to lambda-carrageenan. Cancer Res 1997;57:2823-2826.

12 Tobacman JK, Walters KS: Carrageenan-induced inclusions in mammary myoepithelial cells. Cancer Detect Prev 2001;25:520-526.

13 Zhou G, Sun Y, Xin H, Zhang Y, Li Z, Xu Z: In vivo antitumor and immunomodulation activities of different molecular weight lambda-carrageenans from Chondrus ocellatus. Pharmacol Res 2004;50:47-53.

14 Burger MM: Cell surface alterations in transformed and mitotic normal cells monitored with plant agglutinins. Neoplasma 1973;20: 579-581.

15 Kovař́ík J, Ryšavý O: Lectins in pathology; dissertation thesis, Olomouc, 1984.
16 Bloch R, Jenkins J, Roth J, Burger MM: Purification and charcterization of two lectins from Caragana arborescens seeds. J Biol Chem 1976;251:5929-5935.

17 Thomson AW, Wilson AR, Cruickshank WJ, Horne CWH: Evaluation of carrageenan as an immunosuppressive agent and mediator of intravascular coagulation. Biomedicine 1976;24:102-106.

18 Bekemeier H, Gabor M, Hirshelmann R: Influence of flavonoids and lanthanides on kappa-carrageenin rat tail thrombosis. Exp Pathol 1990;40:61-63.

19 Bekemeier H, Hirshelmann R, Giessler AJ: Carrageenin-induced thrombosis in the rat and mice: a model for testing of antithrombotic substances? Agents Actions 1985;16: 446-451.

20 Hirschelmann R, Bekemeier H: Heparin and phenprocoumon inhibit kappa-carrageenin induced thrombosis in rats. Folia Haematol 1986;113:379-382.

-21 Bertelli A, Bertelli AA, Galmozzi G, Giovanni L, Mian M: Thrombosis induced by endothelin (ET-1) and carrageenin in rats treated with indomethacin and propionyl carnitine. Drugs Exp Clin Res 1993;19:75-78.

22 Nicklin S, Miller K: Effect of orally administered food-grade carrageenans on antibodymediated and cell-mediated immunity in the inbred rat. Food Chem Toxicol 1984;22:615621. 
23 Thomson AW, Fowler EF: Carrageenan: a review of its effects on the immune system. Agents Actions 1981;11:265-273.

24 Winter CA, Risley EA, Nuss CW: Carrageenin-induced edema in hind paw of the rat as an assay for antiiflammatory drugs. Proc Soc Exp Biol Med (NY) 1962;111:544-547.

25 Anderson W, Harthill JE, Zeitlin IJ: Structure-activity in the carrageenans: iota-carrageenan and experimental oedemagenic activity. J Pharm Pharmacol 1984;36:808-813.

26 Bekemeier H, Hirshelmann R, Giessler AJ: Carrageenin-induced thrombosis in the rat and mouse as a test model of substances influencing thrombosis. Biomed Biochim Acta 1984;43:S347-S350.
27 Liang AH, Yu CA, Xue BY, Wang JH, Huang LQ: Comparative research of thrombosis animal model induced by endotoxin in combination with carrageenan in different animals. Zhongguo Zhong Yao Za Zhi 2006;31 57-61.

28 Tandler B, Dunlap M, Hoppel CL, Hassan M: Giant mitochondria in a cardiomyopathic heart. Ultrastruct Pathol 2002;26:177-183.

29 Ghadially FG: Ultrastructural Pathology of the Cell and Matrix, ed 4. Boston, Buterworth-Heinemann, 1997, vol 1, pp 280-293.

30 Bonney RJ, Gery I, Lin TY, Meyenhofer MF, Acevedo W, Davies P: Mononuclear phagocytes from carrageenan-induced granulomas. Isolation, cultivation, and characterization. J Exp Med 1978;48:261-275.

-31 Pugh-Humphreys RG, Thomson AW: An ultrastructural study of peritoneal mononuclear phagocytes from iota carrageenan injected mice. Cytobios 1979;24:211-218.
32 Bowers RR, Stapleton ME, Lew PD: An ultrastructural study of the macrophages of the carrageenan-induced granuloma in the rat lung. J Pathol 1983;140:29-40.

33 Fowler EF, Simpson JG, Thomson AW: Histological and ultrastructural changes following carrageenan injection in the mouse. J Pathol 1980;132:63-79.

34 Calkosinski I, Cegielski M, Dziegel P, Skalik $\mathrm{R}$, Majda J: Apoptotic changes in the myocardium in the course of experimentally-induced pleurisy. Folia Morphol (Warszaw) 2004;63:225-228.

35 Davidson RJ, Simpson JG, Whiting PH, Milton JI, Thomson AW: Haematological changes following systemic injection of purified carrageenans (kappa, lambda and iota). Br J Exp Pathol 1981;62:529-539. 